OPEN ACCESS

Edited by:

Yun-Qing Li,

Fourth Military Medical University,

China

Reviewed by:

Yingwei Mao,

Pennsylvania State University, USA

Zilong Qiu,

Chinese Academy of Sciences, China

Dawen Cai,

University of Michigan, USA

*Correspondence:

$\mathrm{JiHu}$

huji@shanghaitech.edu.cn

Received: 06 August 2016 Accepted: 28 September 2016 Published: 10 October 2016

Citation:

Hu R, Jin S, He X, Xu F and Hu J (2016) Whole-Brain Monosynaptic Afferent Inputs to Basal Forebrain

Cholinergic System.

Front. Neuroanat. 10:98.

doi: 10.3389/fnana.2016.00098

\section{Whole-Brain Monosynaptic Afferent Inputs to Basal Forebrain Cholinergic System}

\author{
Rongfeng $\mathrm{Hu}^{1}$, Sen $\mathrm{Jin}^{2}$, Xiaobin $\mathrm{He}^{2}$, Fuqiang $\mathrm{Xu}^{2}$ and $\mathrm{Ji} \mathrm{Hu}$ \\ ${ }^{1}$ Center for Neuron and Disease, Frontier Institute of Science and Technology, Xi'an Jiaotong University, Xi'an, China, \\ ${ }^{2}$ Center for Excellence in Brain Science, Wuhan Institute of Physics and Mathematics, Chinese Academy of Sciences, \\ Wuhan, China, ${ }^{3}$ School of Life Science and Technology, ShanghaiTech University, Shanghai, China
}

The basal forebrain cholinergic system (BFCS) robustly modulates many important behaviors, such as arousal, attention, learning and memory, through heavy projections to cortex and hippocampus. However, the presynaptic partners governing BFCS activity still remain poorly understood. Here, we utilized a recently developed rabies virus-based cell-type-specific retrograde tracing system to map the whole-brain afferent inputs of the BFCS. We found that the BFCS receives inputs from multiple cortical areas, such as orbital frontal cortex, motor cortex, and insular cortex, and that the BFCS also receives dense inputs from several subcortical nuclei related to motivation and stress, including lateral septum, central amygdala, paraventricular nucleus of hypothalamus, dorsal raphe, and parabrachial nucleus. Interestingly, we found that the BFCS receives inputs from the olfactory areas and the entorhinal-hippocampal system. These results greatly expand our knowledge about the connectivity of the mouse BFCS and provided important preliminary indications for future exploration of circuit function.

Keywords: basal forebrain cholinergic system, whole-brain mapping, monosynaptic inputs, rabies viruses, learning and memory

\section{INTRODUCTION}

Acetylcholine is very important for brain function and has the ability to reconfigure the brain network drastically (Bargmann, 2012). The basal forebrain cholinergic system (BFCS) has been related to brain states and believed to regulate several important behaviors, remarkably involving sleep, attention, decision making (Kilgard, 2003; Yeomans, 2012; Chubykin et al., 2013; Irmak and De Lecea, 2014; Shi et al., 2015). The dysfunction of BFCS is related to neurological disorders such as Alzheimer's disease (AD) and schizophrenia (Cuello et al., 2010; Bohnen and Albin, 2011; Marra et al., 2012; Banuelos et al., 2013; Burke et al., 2013). Overall, BF nuclei show heterogeneous (Zaborszky et al., 2012). Different type of BF neurons have variable physiological properties and response dynamics to stimuli, representing their specific repertoire of afferent inputs (Hangya et al., 2015).

Abbreviations: ACC, anterior cingulate cortex; AMYG, amygdala; BNST, bed nuclei of the stria terminalis; $\mathrm{CPu}$, caudate putamen; CxA, cortex-amygdala transition; DRN, dorsal raphe nucleus; IC, insular cortx; LDT, lateraldorsal tegmental nucleus; LEnt, lateral entorhinal cortex; LH, lateral hypothalamus; M1/2, motor cortex; mPFC, medial prefrontal cortex; NAc, nucleus accumbens; OFC, orbital frontal cortex; PAG, periaqueductal gray; PBN, parabrachial nucleus; PC, piriform cortex; PVN, paraventricular nucleus of the hypothalamus; PVT, paraventricular thalamus; SON, supraoptic nucleus; vHPC, ventral hippocampus; VP, ventral pallidum; VTA, ventral tegmental area. 
The BFCS makes up a minority of the cell population in the BF (Duque et al., 2007; Wu et al., 2014), in addition to the glutamatergic, GABAergic, and peptidergic neurons (Woolf et al., 1986; Zaborszky et al., 1986, 2012; Depboylu et al., 2012). Traditional tracing approaches have been important in the classification of the major afferents inputs to the $\mathrm{BF}$ (Zaborszky et al., 1991). Nevertheless, a full understanding of whole-brain inputs to the BFCS has been hampered because of the inherent limitations of conventional tracing methods. For example: (1) they cannot distinguish the cell types, such as cholinergic or non-cholinergic cells in the BF; (2) passage fibers through or near target structures may absorb the tracers and this may cause non-specificity; and (3) the efficiency of traditional tracers are relatively low. Therefore, in order to overcome these limitations and map the whole-brain afferent inputs to the BFCS, it is necessary to adopt the modern viral tracing system.

Recently, the genetically modified rabies virus has been used to map the monosynaptic inputs to a genetically defined neuronal subtype (Wickersham et al., 2007). It could be applied to characterize the whole-brain presynaptic parterres of a specific type of neurons within a complicated neural network (Wickersham et al., 2007; Wall et al., 2013; Ogawa et al., 2014; Pollak Dorocic et al., 2014; Weissbourd et al., 2014; Grealish et al., 2015). Here, we utilized such viral tracing system to map the whole-brain afferent inputs of the BFCS and discovered that it directly integrates information from several important nuclei. We identified the direct synaptic inputs from neocortex and olfactory areas in the regulation of cholinergic neurons. Also, we discovered that entorhinal-hippocampal system directly project to the BFCS. In addition, we described the presence of direct afferent inputs from several subcortical circuits which are important in regulation of motivation and stress, including lateral septum (LS), central amygdala (CeA), paraventricular nucleus of hypothalamus (PVH), DRN, and PBN. Our findings should be valuable to guide the further investigation of the functional role of the BFCS underlying the normal and neurological disease conditions.

\section{MATERIALS AND METHODS}

\section{Animals}

All procedures were approved by Institutional Animal Care and Use Committees. Experiments were performed exactly as approved by the IACUC at ShanghaiTech University and Center for Excellence in Brain Science, Wuhan Institute of Physics and Mathematics, the Chinese Academy of Sciences, China. The BAC-transgenic ChAT-Cre mice, obtained from MMRRC (Davis, CA, USA), was utilized throughout the study. These mice were back crossed with the C57BL/6J mice. And the obtained mice were used in the study. The mice were raised under $12 / 12$ day/night cycle at the temperature of $22-25^{\circ} \mathrm{C}$, with ad libitum access to rodent chow and water freely available in environmentally controlled conditions. Four adult mice of either sex (2-3 months old) were used in the study, and we also used the wild-type C57BL/6J mice wild-type C57BL/6J mice as control.

\section{Viruses and Surgery}

AAV-CAG-DIO-TVA-GFP (AAV2/9, $1.7 \times 10^{13}$ genomic copies per $\mathrm{ml}$ ), AAV-CAG-DIO-RG (AAV2/9, $6.8 \times 10^{12}$ genomic copies per $\mathrm{ml}$ ), and EnvA-pseudotyped, glycoprotein (RG)deleted and DsRed-expressing rabies virus (RV-EvnA-DsRed, RV; $5.0 \times 10^{8}$ genomic copies per $\mathrm{ml}$ ) were packaged and provided by F. Xu' Lab (Wuhan, China). Briefly, the initial rabies viruses SAD$1 G$ - mCherry (EnvA) and the rabies propagation and titering cell lines were both provided by E.M. Callaway at Salt Institute. The detailed production and concentration procedures for modified rabies viruses were previously described (Osakada et al., 2011). Cre-dependent helper virus vectors, AAV-CAG-DIO-TVA-GFP, and AAV-CAG-DIO-RG were also produced for retrograde transsynaptic tracing (Wang et al., 2015). The CAG promoter of these two plasmids were sub-cloned from AAV-CAG-GFPires-CRE plasmid (Addgene plasmid 48201). And the coding region of TVA-GFP and RG were obtained from the AAV-EF1aFLEX-GT plasmid (Addgene plasmid 26198) and were separately constructed into the DIO cassette of the plasmid pAAV-EF1aDIO-hChR2 (H134R)-EYFP (Addgene plasmid 20298).

Surgery procedures were generally followed by previous studies (Liu et al., 2014; Hu et al., 2016). Briefly, mice were anesthetized by intraperitoneal injection of atropine $(0.05 \mathrm{mg} / \mathrm{kg})$ and pentobarbital $(80 \mathrm{mg} / \mathrm{kg})$, kept warm $\left(37^{\circ} \mathrm{C}\right)$ with an electric heating pad (BrainKing Biotech), and mounted in a stereotaxic holder in order to adjust the skulls of experimental mice parallel with the reference panel. Using a microsyringe pump (Nanoliter, 2000 Injector, WPI), 50-100 $\mathrm{nl}$ of AAV-CAG-DIO-TVA-GFP and AAV-CAG-DIO-RG were stereotaxically injected $\left(46 \mathrm{nl} \mathrm{min}{ }^{-1}\right.$ ) into the unilateral BF (AP: $+0.1 \mathrm{~mm}, \mathrm{ML}:+1.25 \mathrm{~mm}, \mathrm{DV}$ : $5.3 \mathrm{~mm}$ ), followed by an additional $5 \mathrm{~min}$ to allow diffusion of viral particles away from the injection site before slowly withdrawn. Two weeks later, $300 \mathrm{nl}$ of EnvA-pseudotyped, glycoprotein (RG)-deleted and DsRed-expressing rabies virus (RV-EvnA-DsRed, RV; $5.0 \times 10^{8}$ genomic copies per ml) was injected into the same area.

\section{Histology and Image Analysis}

One week after injection of rabies virus, mice were perfused with PBS followed by $4 \%$ paraformaldehyde (PFA) in PBS. After $24 \mathrm{~h}$ of post-fixation in 4\% PFA, coronal brain slices at $60 \mu \mathrm{m}$ thickness were prepared using a cryostat (Leica CM1900). Every third section was counterstained with DAPI (Molecular Probes, Eugene, OR, USA) and visualized with an Olympus VS120 microscope. Further data analyses were performed using Olympus analysis software and ImageJ software. For quantifications of subregions, boundaries were based on the Allen Institute's reference atlas (Lein et al., 2007). We selectively analyzed the retrogradely labeled dense areas. The proportion of total inputs was shown in (Figure 3) and the input densities were expressed as cells $/ \mathrm{mm}^{2}$ (Figure 3).

To characterize the inputs from the orbitofrontal cortex and PC, we immunostained the brain slices containing the two areas with the antibody anti-parvalbumin (PV) according to the previous study (Hu et al., 2016). Briefly, the sections were first blocked with $3 \%$ BSA in PBS- $0.3 \%$ Triton X-100 for $30 \mathrm{~min}$ 


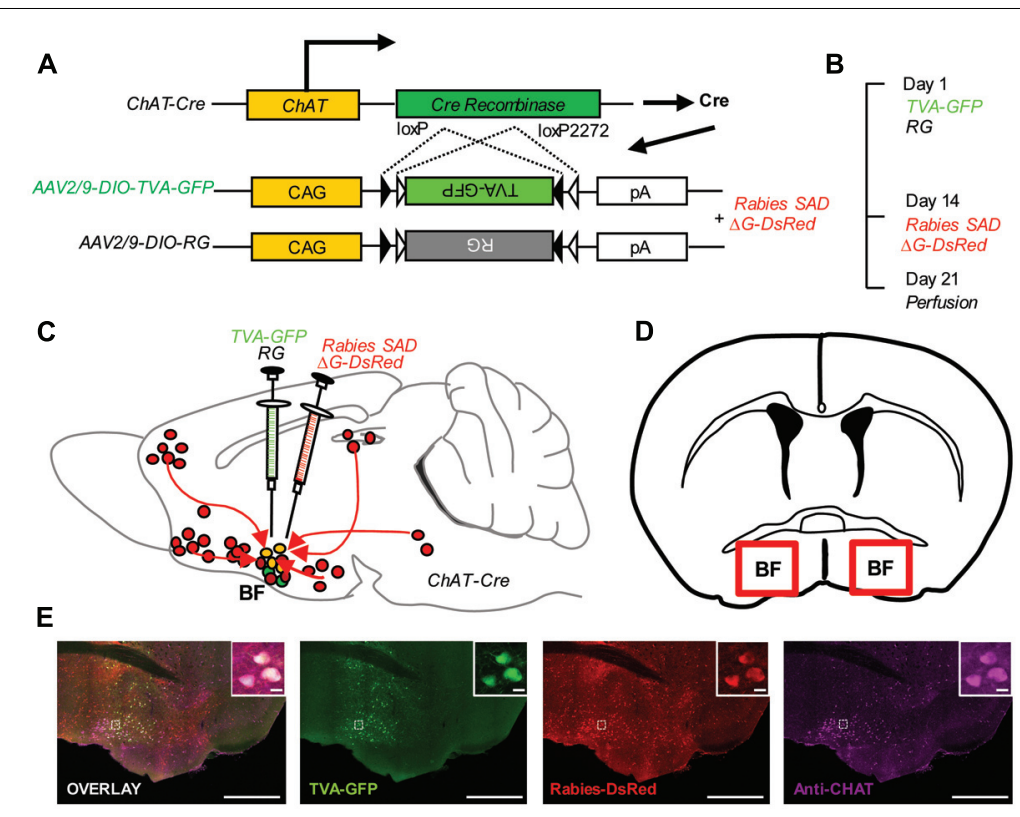

FIGURE 1 | Monosynaptic inputs to the BFCS using rabies-based transsynaptic tracing approach. (A) AAV helper viruses with Cre-dependent expression of TVA receptor and RG. Genetically modified rabies virus is pseudotyped with EnvA. The RG gene is replaced by DsRed. (B) Experimental timeline. (C,D) Sagittal section (C) and coronal section (D) for schematic illustration of AAV helper virus (green) and rabies virus (red) injection into the BF of ChAT-Cre mice. (E) Example of injection site: scale bar, $2 \mathrm{~mm}$. Zoom-in view of injection site: scale bar, $10 \mu \mathrm{m}$.

and incubated with the primary rabbit anti-PV antibody $(1: 200$, Abcam) for $48 \mathrm{~h}$ at $4^{\circ} \mathrm{C}$. After washing, the sections were incubated with second antibody (Alexa Fluor 488 goat antirabbit, Abcam; 1:1000, $2 \mathrm{~h}$ ) at room temperature. Imaging and analysis of images were carried out with a digital slide scanner (CarlZeiss, LSM710).

\section{RESULTS}

\section{Identification of Monosynaptic Inputs onto the BF Cholinergic Neurons Using a Rabies-Based System}

We genetically targeted the BFCS based on a transgenic mouse line expressing Cre recombinase in cholinergic neurons (ChATCre mice) (Gong et al., 2007). In ChAT-Cre mice, we applied a genetically engineered viral system to map the whole-brain afferent inputs to the BFCS. We first co-expressed the avian receptor TVA and the rabies glycoprotein G (RG) in the BFCS neurons, which is achieved through injection of two AAVDIO helper viruses (AAV-DIO-TVA-GFP and AAV-DIO-RG; Figure 1A) into the BF (Figures 1C,D) of ChAT-Cre mice (Cardin et al., 2009). Two weeks later, we injected the genetically modified rabies virus with the avian virus envelope protein (EnvA) (Figures 1A-C). In addition, the endogenous RG of this rabies virus has been genetically substituted to express the fluorescent protein DsRed (Figure 1A). So this rabies virus can only infect cells expressing the TVA receptor, which should be restricted to Cre-expressing BFCS neurons (Figure 1E, Supplementary Figure S1). TVA-GFP signals were colocalized with CHAT immunoreactivity in the majority ( $90 \%)$ of the neurons in the BF (Figure 1E, Supplementary Figure S1), confirming the accuracy of CHAT driver mouse lines. Taken together, we could use this modern technique to map the wholebrain monosynaptic afferent inputs to the BFCS (Figure 1E).

\section{Overview of the Whole-Brain Inputs to BFCS Neurons}

To outline the whole-brain monosynaptic afferent inputs to the BFCS, we explored serial coronal brain sections (Figure 2, Supplementary Figure S2) after viral tracing. Sections from typical ChAT-cre (Figure 2, Supplementary Figure S2) brains showed that DsRed ${ }^{+}$afferent input neurons are largely located in ipsilateral forebrain and midbrain nuclei. We found that the BFCS neurons integrate inputs from a wider than previously believed brain regions. For example, the BFCS received direct synaptic inputs from neocortex, olfactory areas, entorhinalhippocampal system directly project to the BFCS. Also, we found that many motivation and stress related subcortical nuclei provided direct input to the BFCS, such as the LS, CeA, PVH, DRN, and PBN. Finally, many regions of the brain were sparsely labeled, including thalamus and some hindbrain nuclei. To describe the whole-brain distribution of the afferent inputs of the BFCS, we allocated each brain into 22 areas of interest and then counted the input numbers and calculated the input densities (Figure 3). We also carried out the immunostaining experiments to characterize the inputs from the orbitofrontal cortex (OFC) and PC, and the results showed that the neurons in the OFC and PC that target the BFCS are not parvalbumin-positive neurons (Supplementary Figure S3). 

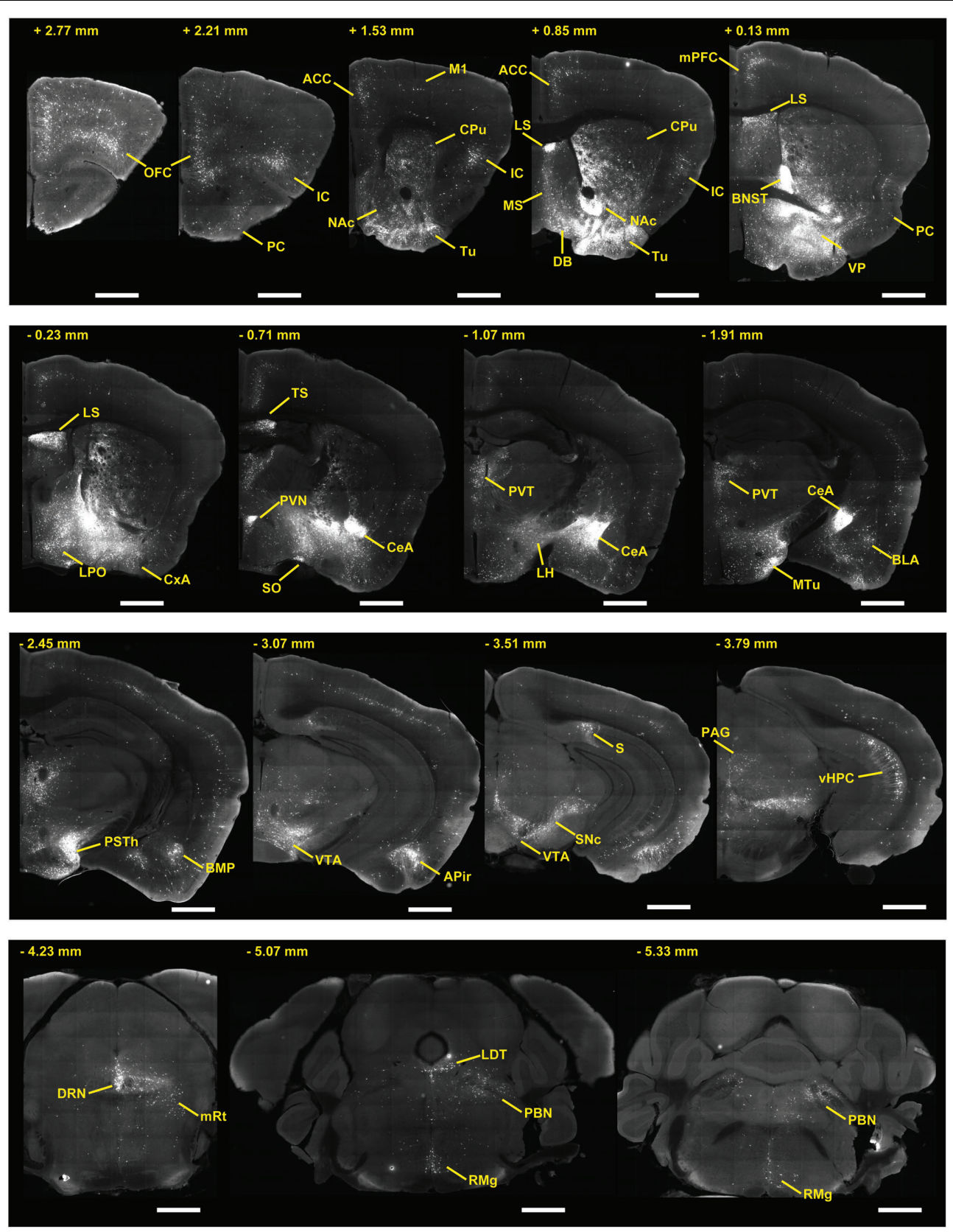

Abbreviations used are the following: OFC, orbital frontal cortex; IC, insular cortx; ACC, anterior cingulate cortex; M1, primary motor cortex; CPu, caudate putamen; Tu, olfactory tubercle; DB, diagonal band; NAc, nucleus accumbens; MS, medial septum; PC, piriform cortex; LS, lateral septal nucleus; mPFC, medial prefrontal cortex; BNST, bed nuclei of the stria terminalis; VP, ventral pallidum; LPO, lateral preoptic area; TS, triangular septal nucleus; PVT, paraventricular thalamus; PVN, paraventricular nucleus; SON, supraoptic nucleus; $\mathbf{C x A}$, cortex-amygdala transition; CeA, central amygdalar nucleus; $\mathbf{L H}$, lateral hypothalamus; BLA, basolateral amygdala; MTu, medial tuberal nucleus; PSTh, parasubthalamic nucleus; BMP, basomedial amygdaloid nu, post; S, subiculum; VTA, ventral tegmental area; APir, amygdalopiriform transition area; SNc, substantia nigra, compact part; vHPC, ventral hippocampus; PAG, periaqueductal gray; LDT, lateraldorsal tegmental nucleus; DRN, dorsal raphe nucleus; mRt, mesencephalic reticular formation; PBN, parabrachial nucleus; $\mathbf{R M g}$, raphe magnus nucleus.

FIGURE 2 | Representative coronal sections showing labeling of monosynaptic inputs to the BFCS. Scale bar: $1 \mathrm{~mm}$. 


\section{Proportion of total inputs (\%) Cell densities (cells $/ \mathrm{mm}^{2}$ )}

\begin{tabular}{|c|c|c|c|c|}
\hline \multirow{4}{*}{ Cortex } & \multirow{2}{*}{$\begin{array}{l}\text { Orbital } \\
\text { Motor }\end{array}$} & \multirow{2}{*}{ 다 } & \multicolumn{2}{|c|}{ Orbital } \\
\hline & & & Motor & 1 \\
\hline & Insular & a & Insular & a \\
\hline & Medial prefrontal cortex & a & Medial prefrontal cortex & 1 \\
\hline \multirow{4}{*}{$\begin{array}{l}\text { Olfactory } \\
\text { areas }\end{array}$} & Olfactory bulb & $\pi$ & Olfactory bulb & \\
\hline & Anterior olfactory nucleus & I & Anterior olfactory nucleus & \\
\hline & Piriform & - & Piriform & I \\
\hline & Olfactory tubercle & - & Olfactory tubercle & $\Rightarrow$ \\
\hline \multirow{2}{*}{ Striatum } & Caudoputamen & =म & Caudoputamen & $=\overline{1}$ \\
\hline & Nucleus Accumbens & 다다 & Nucleus Accumbens & $\Longrightarrow$ \\
\hline \multirow{2}{*}{ Septum } & Lateral septem & $=$ & Lateral septem & 可 \\
\hline & Medial septal nucleus & $=$ & Medial septal nucleus & 모 \\
\hline \multirow{3}{*}{ Hypothalamus } & Lateral Hypothalamus & = & Lateral Hypothalamus & $\bar{\sigma}$ \\
\hline & Supraoptic nucleus & a & Supraoptic nucleus & 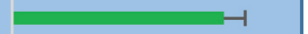 \\
\hline & Paraventricular nucleus & $=1$ & Paraventricular nucleus & $\longrightarrow$ \\
\hline \multirow{2}{*}{ Aymgdala } & Central Amygdala & $\mathrm{CH}$ & Central Amygdala & 담 \\
\hline & Nucleus of the Stria terminal & $|s|$ & Bed Nucleus of the Stria terminal & Us! \\
\hline \multirow[t]{2}{*}{ Thalamus } & Periventricular thalamus & 머 & Periventricular nucleus & 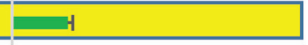 \\
\hline & VentraL tegmental area & 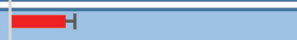 & VentraL tegmental area & $F$ \\
\hline \multirow[t]{2}{*}{ Midbrain } & Dorsal raphe nucleus & - & Dorsal raphe nucleus & 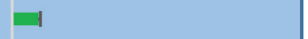 \\
\hline & Periaqueductal gray & 모 & Periaqueductal gray & 1 \\
\hline \multirow[t]{2}{*}{ Pons } & Parabrachial nucleus & - & Parabrachial nucleus & - \\
\hline & & 10 & 20 & 1000 \\
\hline
\end{tabular}

FIGURE 3 | Statistical analysis of the monosynaptic inputs (Left) Proportion of input neurons for BF cholinergic neurons. The values are the normalized ratio of the cell number in each area against the total number of input neurons. Error bars represent the SEM. $n=4$ ChAT-Cre mice. (Right) Cell density of input neurons in each brain area.

\section{BFCS Neurons Receive Widespread Cortical Inputs}

The BFCS has been suggested to control cortical states through projections across the whole neocortical areas (Sarter and Bruno, 2000; Pinto et al., 2013; Kimura et al., 2014; Ljubojevic et al., 2014). Also, there was evidence demonstrating that the $\mathrm{BF}$ received input back from the cortex (Zaborszky et al., 1997). However, the detailed organizations of cortical areas which monosynaptically target BF neurons was uncharacterized, especially in a cell-type-specific manner. Here, we found that across the neocortical areas, the most abundant labeling was found in the ipsilateral anterior cortex, including OFC, insular cortex (IC), primary motor cortex (M1), mPFC, and frontal association (Figures 2 and 4A), demonstrating that BFCS is one of the direct targets of the anterior cortexes (Figure 3). In contrast, sparse labeling was found in the posterior neocortical areas. To our surprise, nearly all posterior neocortical areas project to the BFCS, which most of the retrograde labeled neurons are located at layer 5 (Figure 2, Supplementary Figure S2). The bidirectional connectivity between the cortexes and the $\mathrm{BF}$ has attracted great interest as a circuit involved in modulating decision making, cortical arousal, and learning and memory (Ramanathan et al., 2009; Martinowich et al., 2012; Paolone et al., 2012; Bloem et al., 2014). Our results should provide the foundations for further investigation of circuit mechanism of those important behaviors.

\section{BFCS Neurons Receive Widespread Olfactory Inputs}

Olfactory cues are essential for animal survival (Ligout et al., 2004; Asahina et al., 2008). Here, we found that the BFCS received significant monosynaptic inputs from olfactory areas, including olfactory bulb (OB), anterior olfactory nucleus (AON) and PC (Figures 2 and 3). Among them, the $\mathrm{PC}$ is the largest inputs to the BFCS neurons. The PC is divided into anterior (aPC) and posterior $(\mathrm{pPC})$ portions, and the former receives more afferent input from the $\mathrm{OB}$ and fewer associational inputs, and links more strongly to the outside world (Bekkers and Suzuki, 2010). Our results revealed that both aPC and pPC both project to the BFCS neurons. The OB sends fewer projections to the BFCS neurons, mainly from the Mitral/Tufted cell layer. The AON plays a pivotal but relatively poorly understood role in the processing of odor information. We observed that many AON neurons sent direct inputs onto the BFCS neurons, which may provide the new perspectives for the functional role of AON.

\section{BFCS Neurons Receive Inputs from Entorhinal-Hippocampal System}

The entorhinal-hippocampal system, which consists of the hippocampus, perirhinal cortex, the dentate gyrus, the subicular areas and the entorhinal cortex (EC), is one of the most important brain network for memory and emotion (Buzsaki and Moser, 2013). It plays an important role in declarative 

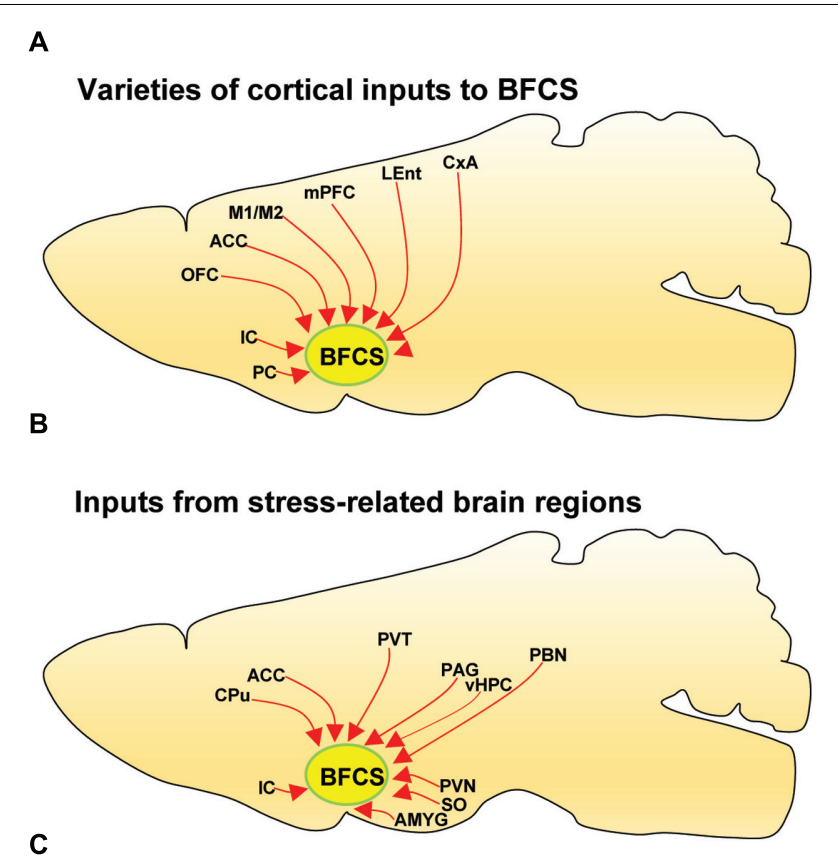

Inputs from motivation-related brain regions

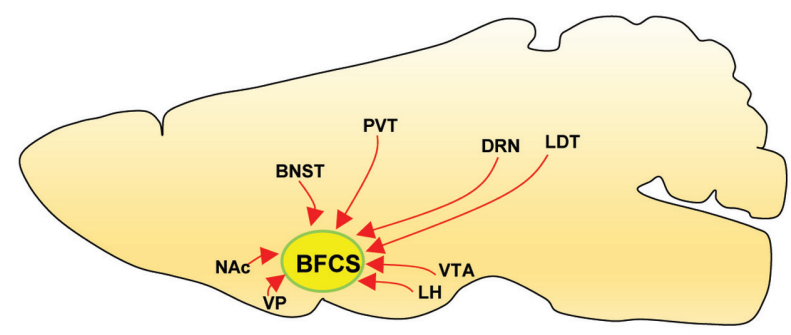

FIGURE 4 | Summarized connectivity of the BFCS. (A) Schematic summary of cortical inputs to the BFCS. (B) Schematic summary of inputs from stress-related brain regions to the BFCS. (C) Schematic summary of inputs from motivation-related brain regions to the BFCS.

memories and in particular spatial memories including memory formation, memory consolidation, and memory optimization in sleep (Buzsaki and Moser, 2013). Here, we found that the EC and vHPC sent strong projections to the BFCS neurons. Notably, the BFCS neurons received dense inputs from the dorsal subiculum (Figure 2, Supplementary Figure S2). These results suggested that there was crosstalk between cholinergic system and hippocampal system, which should be considered in the future investigation of the role of acetylcholine in learning and memory and emotion.

\section{Multiple Stress-Related Brain Areas Target BFCS}

Stress has been implicated in the emergence of numerous pathologies, including depression and anxiety disorders, and as a behavioral modifier that affects fundamental processes, such as learning and memory (Bains et al., 2015; Hollon et al., 2015). Interestingly, our results demonstrated that the BFCS receives strong inputs from many stress-related brain areas (Figures 2, 3, and $4 \mathrm{~B}$ ). One of the prominent retrograde labeled brain region is the amygdala. The amygdala has long been associated with emotion (Janak and Tye, 2015). Understanding the intricacies of amygdala circuitry is of tremendous importance given that the amygdala is implicated in a wide range of disease states, including addiction, autism and anxiety disorders (Janak and Tye, 2015). Conventional retrograde tracing studies have determined that projections from amygdala to BF originate primarily from the $\mathrm{CeA}$, with limited inputs from the other amygdala nuclei. Both the central and basolateral nuclei of the amygdala are known to innervate $\mathrm{BF}$, but the specificity and relevance of this input stream is not well understood. In accordance with previous studies, we detected the majority of DsRed-labeled amygdala input neurons in the CeA (Figures 2 and 3), and we just found few DsRed-labeled neurons in basolateral amygdala (BLA) and medial amygdala nucleus (MeA) (Figures 2 and 3, Supplementary Figure S2).

In addition to amygdala, there are several other stress-related brain regions sending strong projections to the BFCS, such as LS, PVT, vHPC, PVH, SON, periaqueductal grey matter (PAG), and $\mathrm{PBN}$. These structures are involved in the autonomic, hormonal and behavioral responses to stress and fear. Our results may provide insights for the further investigation of circuit mechanism of depression and anxiety disorder.

\section{Motivation-Related Brain Regions Send Projections to BFCS}

The VP is a crucial node in the ventral striatopallidal circuits underlying reward-related behaviors, with major reciprocal connections to the NAc (Mahler et al., 2014). Our monosynaptic tracing data showed that the VP and NAc both strongly target the BFCS (Figures 2 and $4 \mathrm{C}$ ). The bed nucleus of the stria terminalis (BNST), a neural component of the extended amygdala (Jennings et al., 2013), is a key integrator of diverse motivational states through its interactions with various synaptic targets, including the $\mathrm{LH}$, a brain region historically implicated in reward and motivation (Harris et al., 2005). Interestingly, our results demonstrated that these two brain areas densely targeted the BFCS (Figures 2 and 4C), indicating that BFCS may be regulated by motivational events. Also, the limbic system, including limbic amygdala, conveys affective value information to the BFCS (Figure 2). Both traditional tracing techniques and rabies virusbased monosynaptic tracing approaches have discovered that BF neurons project their axons into the brain regions controlling the reward system, including VTA, DRN, and LDT (Zaborszky et al., 2012). Here, our results showed that these brain areas also send dense projections to the BFCS (Figures 2 and 4C), indicating that the BFCS bi-directionally connects multiple motivation-related brain regions to modulate the behaviors.

\section{DISCUSSION}

The BFCS is essential to some of the most vital functions of the brain, including attention, arousal, decision making, and learning and memory. To understand the circuit mechanism of how the BFCS modulate this broad spectrum of behavior, it is 
crucial to explore the afferent inputs impacting the activity of the BFCS (Alheid, 2003). Traditional mapping of inputs to the BF was based on conventional retrograde tracing techniques, which does not allow for identification of specific afferent of cholinergic neurons (Wall et al., 2013; Ogawa et al., 2014; Pollak Dorocic et al., 2014; Weissbourd et al., 2014). In the present study, our rabies-based system efficiently mapped the extensive whole-brain afferent inputs of the BFCS, especially inputs from a wide range of cortical regions, olfactory areas, entorhinal-hippocampal system, stress-related brain regions, and motivation-related brain nuclei (Figures 2-4). Comparing to previous results using traditional methods, our findings are more precise and efficiency, which should provide a comprehensive map of the presynaptic partners that control the BFCS.

\section{Implications for the Role of BFCS in Attention and Decision Making}

Basal forebrain cholinergic system has long been implicated in attention (Dalley et al., 2004; Martinez and Sarter, 2004; Sarter et al., 2005, 2014; Sarter, 2007; Broussard et al., 2009; Fadel and Burk, 2010; Paolone et al., 2012; D’Souza and Vijayaraghavan, 2014; Ljubojevic et al., 2014; Oros et al., 2014; Parikh et al., 2014), an important behavioral and cognitive process through selectively concentrating on a discrete aspect of information (Paolone et al., 2012). Previous studies showed that animals with restricted BFCS lesion causes disruption in attentional processing (Muir et al., 1992a,b, 1993, 1994; McGaughy and Sarter, 1999; Waite et al., 1999; Dalley et al., 2004). The higherorder association cortices in the temporal and parietal lobes and prefrontal cortex (PFC) also have been found to mediate aspects of attention (Craft et al., 2005). In addition, previous findings showed PFC areas support appropriate decision-making (Kennerley and Walton, 2011; Steiner and Redish, 2014; Siegel et al., 2015). Our result revealed that BFCS is one of the major target of PFC. These two areas are interconnected to support attentional processing. Cortical cholinergic neurotransmission is regulated on multiple timescales to mediate the detection of behaviorally significant cues and to support cognitive performance (Parikh et al., 2007).

\section{Implications for the Role of BFCS in Sleep and Arousal}

Arousal is considered to be play an important role in the pathophysiology of sleep disorders. The BFCS is recognized as important site of sleep-wake regulation (Koyama, 2012; Kalinchuk et al., 2015). The BFCS heavily projects to the entire cortex and limbic system, excites the target cells and involves in the regulation of waking and REM sleep (Jones, 1993; Rodrigo-Angulo et al., 2008; Platt and Riedel, 2011). Optogenetic activation of BFCS induce sleep-awake transition (Han et al., 2014). Here, we showed that BFCS receive widespread input from hypothalamic nuclei, including the preoptic areas and $\mathrm{LH}$, and both have implicated in sleep and arousal regulations (Szymusiak et al., 2007). Our result may provide explanations for how BFCS participate the neural network for brain state and sleep regulation.

\section{Implications for the Role of BFCS in Reward System}

Lesion and stimulation of the VTA increases cholinergic activity in the rat brain (Majkutewicz et al., 2010). Both traditional tracing technique and rabies virus-based monosynaptic tracing approach have discovered that BF neurons project their axons into the brain regions controlling the reward system, including VTA, DRN, NAc, and OFC (Zaborszky et al., 2012). Here, we found that these brain areas also send projections to the BFCS. These collective data indicate that the BF interconnects with the brain reward system, implying that the BFCS is an important node in this system. Also, increasing evidence indicated that the VP, a subregion of the BF, plays vital roles in reward and motivation [see reviewed in Smith et al. (2009)].

Collectively, our viral tracing results provide new perceptive for the future exploration of circuit mechanism underlying the function of the BFCS, such as: (1) The function of olfactory input (directly from OB, AON, Tu, and PC) to the BFCS; (2) The function of feedback cortical projections onto the BFCS. The BFCS plays important role in cortical activation, it should be interesting to examine how cortical neurons (particularly, layer 5 neurons) impact the BFCS; (3) The physiological and behavioral function of entorhinal-hippocampus projections to the BFCS; (4) The comprehensive functions of the BFCS in motivation and reward; (5) Considering the dense inputs from stress-related regions, it should be important to examine the role of BFCS in stress and emotion regulation. Therefore, further functional and behavioral evidence is of great need and interest to be provided.

\section{AUTHOR CONTRIBUTIONS}

$\mathrm{JH}$ and $\mathrm{RH}$ conceptualized the project. $\mathrm{RH}$ performed the majority of experiments. SJ, XH, and FX provided the viruses used in this study. $\mathrm{JH}$ and $\mathrm{RH}$ analyzed the data and wrote the manuscript with the participation of all other authors.

\section{FUNDING}

This work was supported by the National Program on Key Basic Research Project (973 Program; Grant No.2014CB548200 to JH) and the National Natural Science Foundation of China (Grant No. 91432113 to JH).

\section{ACKNOWLEDGMENT}

We thank all members of Dr. Minmin Luo' lab at National Institute of Biological Sciences, Beijing.

\section{SUPPLEMENTARY MATERIAL}

The Supplementary Material for this article can be found online at: http://journal.frontiersin.org/article/10.3389/fnana.2016. 00098 


\section{REFERENCES}

Alheid, G. F. (2003). Extended amygdala and basal forebrain. Ann. N. Y. Acad. Sci. 985, 185-205. doi: 10.1111/j.1749-6632.2003.tb07082.x

Asahina, K., Pavlenkovich, V., and Vosshall, L. B. (2008). The survival advantage of olfaction in a competitive environment. Curr. Biol. 18, 1153-1155. doi: 10.1016/j.cub.2008.06.075

Bains, J. S., Cusulin, J. I. W., and Inoue, W. (2015). Stress-related synaptic plasticity in the hypothalamus. Nat. Rev. Neurosci. 16, 377-388. doi: 10.1038/nrn3881

Banuelos, C., Lasarge, C. L., Mcquail, J. A., Hartman, J. J., Gilbert, R. J., Ormerod, B. K., et al. (2013). Age-related changes in rostral basal forebrain cholinergic and GABAergic projection neurons: relationship with spatial impairment. Neurobiol. Aging 34, 845-862. doi: 10.1016/j.neurobiolaging.2012.06.013

Bargmann, C. I. (2012). Beyond the connectome: how neuromodulators shape neural circuits. Bioessays 34, 458-465. doi: 10.1002/bies.201100185

Bekkers, J. M., and Suzuki, N. (2010). Inhibitory neurons in the anterior piriform cortex of the mouse: classification using molecular markers. J. Comp. Neurol. 518, 1670-1687. doi: 10.1002/cne.22295

Bloem, B., Schoppink, L., Rotaru, D. C., Faiz, A., Hendriks, P., Mansvelder, H. D., et al. (2014). Topographic mapping between basal forebrain cholinergic neurons and the medial prefrontal cortex in mice. J. Neurosci. 34, 16234-16246. doi: 10.1523/JNEUROSCI.3011-14.2014

Bohnen, N. I., and Albin, R. L. (2011). The cholinergic system and Parkinson disease. Behav. Brain Res. 221, 564-573. doi: 10.1016/j.bbr.2009.12.048

Broussard, J. I., Karelina, K., Sarter, M., and Givens, B. (2009). Cholinergic optimization of cue-evoked parietal activity during challenged attentional performance. Eur. J. Neurosci. 29, 1711-1722. doi: 10.1111/j.1460-9568.2009.06713.x

Burke, R. M., Norman, T. A., Haydar, T. F., Slack, B. E., Leeman, S. E., Blusztajn, J. K., et al. (2013). BMP9 ameliorates amyloidosis and the cholinergic defect in a mouse model of Alzheimer's disease. Proc. Natl. Acad. Sci. U.S.A. 110, 19567-19572. doi: 10.1073/pnas.1319297110

Buzsaki, G., and Moser, E. I. (2013). Memory, navigation and theta rhythm in the hippocampal-entorhinal system. Nat. Neurosci. 16, 130-138. doi: 10.1038/nn.3304

Cardin, J. A., Carlen, M., Meletis, K., Knoblich, U., Zhang, F., Deisseroth, K., et al. (2009). Driving fast-spiking cells induces gamma rhythm and controls sensory responses. Nature 459, 663-667. doi: 10.1038/nature08002

Chubykin, A. A., Roach, E. B., Bear, M. F., and Shuler, M. G. (2013). A cholinergic mechanism for reward timing within primary visual cortex. Neuron 77, 723735. doi: 10.1016/j.neuron.2012.12.039

Craft, T. K., Mahoney, J. H., Devries, A. C., and Sarter, M. (2005). Microsphere embolism-induced cortical cholinergic deafferentation and impairments in attentional performance. Eur. J. Neurosci. 21, 3117-3132. doi: 10.1111/j.14609568.2005.04136.x

Cuello, A. C., Bruno, M. A., Allard, S., Leon, W., and Iulita, M. F. (2010). Cholinergic involvement in Alzheimer's disease. A link with NGF maturation and degradation. J. Mol. Neurosci. 40, 230-235. doi: 10.1007/s12031-009-9238-Z

Dalley, J. W., Theobald, D. E., Bouger, P., Chudasama, Y., Cardinal, R. N., and Robbins, T. W. (2004). Cortical cholinergic function and deficits in visual attentional performance in rats following 192 IgG-saporin-induced lesions of the medial prefrontal cortex. Cereb. Cortex 14, 922-932. doi: 10.1093/cercor/bhh052

Depboylu, C., Weihe, E., and Eiden, L. E. (2012). Lentiviral infection of rhesus macaques causes long-term injury to cortical and hippocampal projections of prostaglandin-expressing cholinergic basal forebrain neurons. J. Neuropathol. Exp. Neurol. 71, 15-27. doi: 10.1097/NEN.0b013e31823cfac5

D'Souza, R. D., and Vijayaraghavan, S. (2014). Paying attention to smell: cholinergic signaling in the olfactory bulb. Front. Synaptic Neurosci. 6:21. doi: 10.3389/fnsyn.2014.00021

Duque, A., Tepper, J. M., Detari, L., Ascoli, G. A., and Zaborszky, L. (2007). Morphological characterization of electrophysiologically and immunohistochemically identified basal forebrain cholinergic and neuropeptide Y-containing neurons. Brain Struct. Funct. 212, 55-73. doi: 10.1007/s00429-007-0143-3

Fadel, J., and Burk, J. A. (2010). Orexin/hypocretin modulation of the basal forebrain cholinergic system: role in attention. Brain Res. 1314, 112-123. doi: 10.1016/j.brainres.2009.08.046
Gong, S., Doughty, M., Harbaugh, C. R., Cummins, A., Hatten, M. E., Heintz, N., et al. (2007). Targeting Cre recombinase to specific neuron populations with bacterial artificial chromosome constructs. J. Neurosci. 27, 9817-9823. doi: 10.1523/JNEUROSCI.2707-07.2007

Grealish, S., Heuer, A., Cardoso, T., Kirkeby, A., Jonsson, M., Johansson, J., et al. (2015). Monosynaptic tracing using modified rabies virus reveals early and extensive circuit integration of human embryonic stem cell-derived neurons. Stem Cell Rep. 4, 975-983. doi: 10.1016/j.stemcr.2015.04.011

Han, Y., Shi, Y. F., Xi, W., Zhou, R., Tan, Z. B., Wang, H., et al. (2014). Selective activation of cholinergic basal forebrain neurons induces immediate sleep-wake transitions. Curr. Biol. 24, 693-698. doi: 10.1016/j.cub.2014.02.011

Hangya, B., Ranade, S. P., Lorenc, M., and Kepecs, A. (2015). Central cholinergic neurons are rapidly recruited by reinforcement feedback. Cell 162, 1155-1168. doi: 10.1016/j.cell.2015.07.057

Harris, G. C., Wimmer, M., and Aston-Jones, G. (2005). A role for lateral hypothalamic orexin neurons in reward seeking. Nature 437, 556-559. doi: 10.1038 /nature04071

Hollon, N. G., Burgeno, L. M., and Phillips, P. E. M. (2015). Stress effects on the neural substrates of motivated behavior. Nat. Neurosci. 18, 1405-1412. doi: 10.1038/nn.4114

$\mathrm{Hu}, \mathrm{R}$. , Zhang, J., Luo, M., and Hu, J. (2016). Response patterns of GABAergic neurons in the anterior piriform cortex of awake mice. Cereb. Cortex doi: 10.1093/cercor/bhw175 [Epub ahead of print].

Irmak, S. O., and De Lecea, L. (2014). Basal forebrain cholinergic modulation of sleep transitions. Sleep 37, 1941-1951. doi: 10.5665/sleep.4246

Janak, P. H., and Tye, K. M. (2015). From circuits to behaviour in the amygdala. Nature 517, 284-292. doi: 10.1038/nature14188

Jennings, J. H., Rizzi, G., Stamatakis, A. M., Ung, R. L., and Stuber, G. D. (2013). The inhibitory circuit architecture of the lateral hypothalamus orchestrates feeding. Science 341, 1517-1521. doi: 10.1126/science.1241812

Jones, B. E. (1993). The organization of central cholinergic systems and their functional importance in sleep-waking states. Prog. Brain Res. 98, 61-71. doi: 10.1016/S0079-6123(08)62381-X

Kalinchuk, A. V., Porkka-Heiskanen, T., Mccarley, R. W., and Basheer, R. (2015). Cholinergic neurons of the basal forebrain mediate biochemical and electrophysiological mechanisms underlying sleep homeostasis. Eur. J. Neurosci. 41, 182-195. doi: 10.1111/ejn.12766

Kennerley, S. W., and Walton, M. E. (2011). Decision making and reward in frontal cortex: complementary evidence from neurophysiological and neuropsychological studies. Behav. Neurosci. 125, 297-317. doi: $10.1037 / \mathrm{a} 0023575$

Kilgard, M. (2003). Cholinergic modulation of skill learning and plasticity. Neuron 38, 678-680. doi: 10.1016/S0896-6273(03)00327-1

Kimura, R., Safari, M. S., Mirnajafi-Zadeh, J., Kimura, R., Ebina, T., Yanagawa, Y., et al. (2014). Curtailing effect of awakening on visual responses of cortical neurons by cholinergic activation of inhibitory circuits. J. Neurosci. 34, 1012210133. doi: 10.1523/JNEUROSCI.0863-14.2014

Koyama, Y. (2012). [Regulation of sleep and wakefulness through the monoaminergic and cholinergic systems]. Brain Nerve 64, 601-610.

Lein, E. S., Hawrylycz, M. J., Ao, N., Ayres, M., Bensinger, A., Bernard, A., et al. (2007). Genome-wide atlas of gene expression in the adult mouse brain. Nature 445, 168-176. doi: 10.1038/nature05453

Ligout, S., Keller, M., and Porter, R. H. (2004). The role of olfactory cues in the discrimination of agemates by lambs. Anim. Behav. 68, 785-792. doi: 10.1016/j.anbehav.2004.01.008

Liu, Z. X., Zhou, J. F., Li, Y., Hu, F., Lu, Y., Ma, M., et al. (2014). Dorsal raphe neurons signal reward through 5-HT and glutamate. Neuron 81, 1360-1374. doi: 10.1016/j.neuron.2014.02.010

Ljubojevic, V., Luu, P., and De Rosa, E. (2014). Cholinergic contributions to supramodal attentional processes in rats. J. Neurosci. 34, 2264-2275. doi: 10.1523/JNEUROSCI.1024-13.2014

Mahler, S. V., Vazey, E. M., Beckley, J. T., Keistler, C. R., Mcglinchey, E. M., Kaufling, J., et al. (2014). Designer receptors show role for ventral pallidum input to ventral tegmental area in cocaine seeking. Nat. Neurosci. 17, 577-585. doi: $10.1038 / \mathrm{nn} .3664$

Majkutewicz, I., Cecot, T., Jerzemowska, G., Trojniar, W., Jaskulski, M., and Wrona, D. (2010). Lesion and stimulation of the ventral tegmental area increases cholinergic activity in the rat brain. Acta Neurobiol. Exp. 70, 28-39. 
Marra, C., Quaranta, D., Profice, P., Pilato, F., Capone, F., Iodice, F., et al. (2012). Central cholinergic dysfunction measured "in vivo" correlates with different behavioral disorders in Alzheimer's disease and dementia with Lewy body. Brain Stimul. 5, 533-538. doi: 10.1016/j.brs.2011.08.009

Martinez, V., and Sarter, M. (2004). Lateralized attentional functions of cortical cholinergic inputs. Behav. Neurosci. 118, 984-991. doi: 10.1037/07357044.118.5.984

Martinowich, K., Schloesser, R. J., Lu, Y., Jimenez, D. V., Paredes, D., Greene, J. S., et al. (2012). Roles of p75(NTR), long-term depression, and cholinergic transmission in anxiety and acute stress coping. Biol. Psychiatry 71, 75-83. doi: 10.1016/j.biopsych.2011.08.014

McGaughy, J., and Sarter, M. (1999). Effects of ovariectomy, 192 IgG-saporininduced cortical cholinergic deafferentation, and administration of estradiol on sustained attention performance in rats. Behav. Neurosci. 113, 1216-1232. doi: 10.1037/0735-7044.113.6.1216

Muir, J. L., Dunnett, S. B., Robbins, T. W., and Everitt, B. J. (1992a). Attentional functions of the forebrain cholinergic systems: effects of intraventricular hemicholinium, physostigmine, basal forebrain lesions and intracortical grafts on a multiple-choice serial reaction time task. Exp. Brain Res. 89, 611-622. doi: 10.1007/BF00229886

Muir, J. L., Everitt, B. J., and Robbins, T. W. (1994). AMPA-induced excitotoxic lesions of the basal forebrain: a significant role for the cortical cholinergic system in attentional function. J. Neurosci. 14, 2313-2326.

Muir, J. L., Page, K. J., Sirinathsinghji, D. J., Robbins, T. W., and Everitt, B. J. (1993). Excitotoxic lesions of basal forebrain cholinergic neurons: effects on learning, memory and attention. Behav. Brain Res. 57, 123-131. doi: 10.1016/01664328(93)90128-D

Muir, J. L., Robbins, T. W., and Everitt, B. J. (1992b). Disruptive effects of muscimol infused into the basal forebrain on conditional discrimination and visual attention: differential interactions with cholinergic mechanisms. Psychopharmacology (Berl) 107, 541-550. doi: 10.1007/BF02245269

Ogawa, S. K., Cohen, J. Y., Hwang, D., Uchida, N., and Watabe-Uchida, M. (2014). Organization of monosynaptic inputs to the serotonin and dopamine neuromodulatory systems. Cell Rep. 8, 1105-1118. doi: 10.1016/j.celrep.2014.06.042

Oros, N., Chiba, A. A., Nitz, D. A., and Krichmar, J. L. (2014). Learning to ignore: a modeling study of a decremental cholinergic pathway and its influence on attention and learning. Learn. Mem. 21, 105-118. doi: 10.1101/lm.032433.113

Osakada, F., Mori, T., Cetin, A. H., Marshel, J. H., Virgen, B., and Callaway, E. M. (2011). New rabies virus variants for monitoring and manipulating activity and gene expression in defined neural circuits. Neuron 71, 617-631. doi: 10.1016/j.neuron.2011.07.005

Paolone, G., Lee, T. M., and Sarter, M. (2012). Time to pay attention: attentional performance time-stamped prefrontal cholinergic activation, diurnality, and performance. J. Neurosci. 32, 12115-12128. doi: 10.1523/JNEUROSCI.227112.2012

Parikh, V., Bernard, C. S., Naughton, S. X., and Yegla, B. (2014). Interactions between Abeta oligomers and presynaptic cholinergic signaling: age-dependent effects on attentional capacities. Behav. Brain Res. 274, 30-42. doi: 10.1016/j.bbr.2014.07.046

Parikh, V., Kozak, R., Martinez, V., and Sarter, M. (2007). Prefrontal acetylcholine release controls cue detection on multiple time scales. Neuron 56, 141-154. doi: 10.1016/j.neuron.2007.08.025

Pinto, L., Goard, M. J., Estandian, D., Xu, M., Kwan, A. C., Lee, S. H., et al. (2013). Fast modulation of visual perception by basal forebrain cholinergic neurons. Nat. Neurosci. 16, 1857-1863. doi: 10.1038/nn.3552

Platt, B., and Riedel, G. (2011). The cholinergic system, EEG and sleep. Behav. Brain Res. 221, 499-504. doi: 10.1016/j.bbr.2011. 01.017

Pollak Dorocic, I., Furth, D., Xuan, Y., Johansson, Y., Pozzi, L., Silberberg, G., et al. (2014). A whole-brain atlas of inputs to serotonergic neurons of the dorsal and median raphe nuclei. Neuron 83, 663-678. doi: 10.1016/j.neuron.2014.07.002

Ramanathan, D., Tuszynski, M. H., and Conner, J. M. (2009). The basal forebrain cholinergic system is required specifically for behaviorally mediated cortical map plasticity. J. Neurosci. 29, 5992-6000. doi: 10.1523/JNEUROSCI.023009.2009

Rodrigo-Angulo, M. L., Heredero, S., Rodriguez-Veiga, E., and Reinoso-Suarez, F. (2008). GABAergic and non-GABAergic thalamic, hypothalamic and basal forebrain projections to the ventral oral pontine reticular nucleus: their implication in REM sleep modulation. Brain Res. 1210, 116-125. doi: 10.1016/j.brainres.2008.02.095

Sarter, M. (2007). Cholinergic control of attention to cues guiding established performance versus learning: theoretical comment on Maddux, Kerfoot, Chatterjee, and Holland (2007). Behav. Neurosci. 121, 233-235. doi: 10.1037/0735-7044.121.1.233

Sarter, M., Albin, R. L., Kucinski, A., and Lustig, C. (2014). Where attention falls: increased risk of falls from the converging impact of cortical cholinergic and midbrain dopamine loss on striatal function. Exp. Neurol. 257, 120-129. doi: 10.1016/j.expneurol.2014.04.032

Sarter, M., and Bruno, J. P. (2000). Cortical cholinergic inputs mediating arousal, attentional processing and dreaming: differential afferent regulation of the basal forebrain by telencephalic and brainstem afferents. Neuroscience 95, 933-952. doi: 10.1016/S0306-4522(99)00487-X

Sarter, M., Hasselmo, M. E., Bruno, J. P., and Givens, B. (2005). Unraveling the attentional functions of cortical cholinergic inputs: interactions between signaldriven and cognitive modulation of signal detection. Brain Res. Brain Res. Rev. 48, 98-111. doi: 10.1016/j.brainresrev.2004.08.006

Shi, Y. F., Han, Y., Su, Y. T., Yang, J. H., and Yu, Y. Q. (2015). Silencing of cholinergic basal forebrain neurons using archaerhodopsin prolongs slow-wave sleep in mice. PLoS ONE 10:e0130130. doi: 10.1371/journal.pone.0130130

Siegel, M., Buschman, T. J., and Miller, E. K. (2015). Cortical information flowduring flexible sensorimotor decisions. Science 348, 1352-1355. doi: 10.1126/science.aab0551

Smith, K. S., Tindell, A. J., Aldridge, J. W., and Berridge, K. C. (2009). Ventral pallidum roles in reward and motivation. Behav. Brain Res. 196, 155-167. doi: 10.1016/j.bbr.2008.09.038

Steiner, A. P., and Redish, A. D. (2014). Behavioral and neurophysiological correlates of regret in rat decision-making on a neuroeconomic task. Nat. Neurosci. 17, 995-1002. doi: 10.1038/nn.3740

Szymusiak, R., Gvilia, I., and Mcginty, D. (2007). Hypothalamic control of sleep. Sleep Med. 8, 291-301. doi: 10.1016/j.sleep.2007.03.013

Waite, J. J., Wardlow, M. L., and Power, A. E. (1999). Deficit in selective and divided attention associated with cholinergic basal forebrain immunotoxic lesion produced by 192-saporin; motoric/sensory deficit associated with Purkinje cell immunotoxic lesion produced by OX7-saporin. Neurobiol. Learn. Mem. 71, 325-352. doi: 10.1006/nlme.1998.3884

Wall, N. R., De La Parra, M., Callaway, E. M., and Kreitzer, A. C. (2013). Differential innervation of direct- and indirect-pathway striatal projection neurons. Neuron 79, 347-360. doi: 10.1016/j.neuron.2013.05.014

Wang, D. Q., He, X. B., Zhao, Z., Feng, Q. R., Lin, R., Sun, Y., et al. (2015). Whole-brain mapping of the direct inputs and axonal projections of POMC and AgRP neurons. Front. Neuroanat. 9:40. doi: 10.3389/fnana.2015. 00040

Weissbourd, B., Ren, J., Deloach, K. E., Guenthner, C. J., Miyamichi, K., and Luo, L. Q. (2014). Presynaptic partners of dorsal raphe serotonergic and GABAergic neurons. Neuron 83, 645-662. doi: 10.1016/j.neuron.2014. 06.024

Wickersham, I. R., Lyon, D. C., Barnard, R. J., Mori, T., Finke, S., Conzelmann, K. K., et al. (2007). Monosynaptic restriction of transsynaptic tracing from single, genetically targeted neurons. Neuron 53, 639-647. doi: 10.1016/j.neuron.2007.01.033

Woolf, N. J., Hernit, M. C., and Butcher, L. L. (1986). Cholinergic and non-cholinergic projections from the rat basal forebrain revealed by combined choline acetyltransferase and Phaseolus vulgaris leucoagglutinin immunohistochemistry. Neurosci. Lett. 66, 281-286. doi: 10.1016/03043940(86)90032-7

Wu, H., Williams, J., and Nathans, J. (2014). Complete morphologies of basal forebrain cholinergic neurons in the mouse. Elife 3:e02444. doi: 10.7554/eLife.02444

Yeomans, J. S. (2012). Muscarinic receptors in brain stem and mesopontine cholinergic arousal functions. Handb. Exp. Pharmacol. 208, 243-259. doi: 10.1007/978-3-642-23274-9_11

Zaborszky, L., Carlsen, J., Brashear, H. R., and Heimer, L. (1986). Cholinergic and GABAergic afferents to the olfactory bulb in the rat with special emphasis on the projection neurons in the nucleus of the horizontal limb of the diagonal band. J. Comp. Neurol. 243, 488-509. doi: 10.1002/cne.902430405 
Zaborszky, L., Cullinan, W. E., and Braun, A. (1991). Afferents to basal forebrain cholinergic projection neurons: an update. Adv. Exp. Med. Biol. 295, 43-100. doi: 10.1007/978-1-4757-0145-6_2

Zaborszky, L., Gaykema, R. P., Swanson, D. J., and Cullinan, W. E. (1997). Cortical input to the basal forebrain. Neuroscience 79, 1051-1078. doi: 10.1016/S03064522(97)00049-3

Zaborszky, L., Van Den Pol, A., and Gyengesi, E. (2012). "The basal forebrain cholinergic projection system in mice," in The Mouse Nervous System, eds C. Watson, G. Paxinos, and L. Puelles (Cambridge, MA: Academic Press), 684-718.
Conflict of Interest Statement: The authors declare that the research was conducted in the absence of any commercial or financial relationships that could be construed as a potential conflict of interest.

Copyright $\odot 2016 \mathrm{Hu}$, Jin, He, Xu and Hu. This is an open-access article distributed under the terms of the Creative Commons Attribution License (CC BY). The use, distribution or reproduction in other forums is permitted, provided the original author(s) or licensor are credited and that the original publication in this journal is cited, in accordance with accepted academic practice. No use, distribution or reproduction is permitted which does not comply with these terms. 